hospital, I took the mattress, bedding, dressings and indeed everything combustible which had been about the patient, into the back yard, saturated it with kerosene and burned it. The room was scrubbed, then washed with a solution of carbolic acid and left open and unoccupied for some time. There were other patients in the hospital who had wounds, but none of them became infected.

200 Oakwood Boulevard.

\section{A NEW FIGURE-OF-8 PEDICLE LIGATURE.} BY F. SHIMONEK, M.D. MILWAUKEE, WIS.

The following pedicle ligature is, so far as I know, original:

I have used it with much satisfaction for the last nine months. It is an improvement upon the old

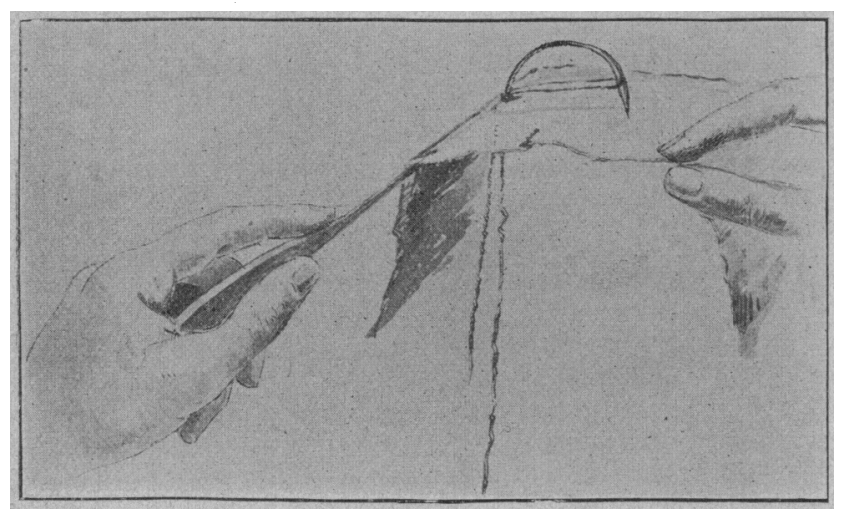

FiguRe 1.

Worllich ligature, because it can be quickly tied, whether it be used singly or as a continuous ligature; for very broad pedicles only one knot is required; the

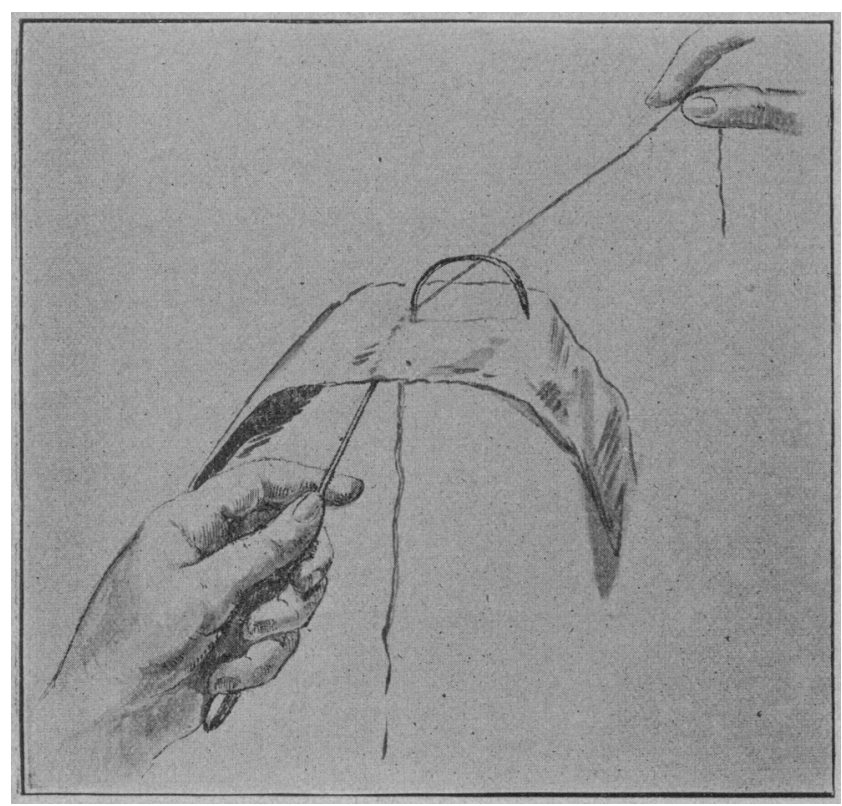

FIGURE 2.

threads cross naturally when passing through the pedicle. It is better than the Tait ligature because of its applicability to any breadth pedicle and can be safely tied, and without any trouble whatever.

An armed needle is passed through the pedicle, as shown in Fig. 1. That part of the ligature passing through the eye of the needle is withdrawn from it, as shown in Fig. 2. We now have the needle and ligature passing through the same opening in the pedicle, and yet are independent of each other. Take that part of the ligature corresponding with the handle of the needle, carry it half way around the pedicle and pass it through the eye of the needle as shown in Fig. 3.

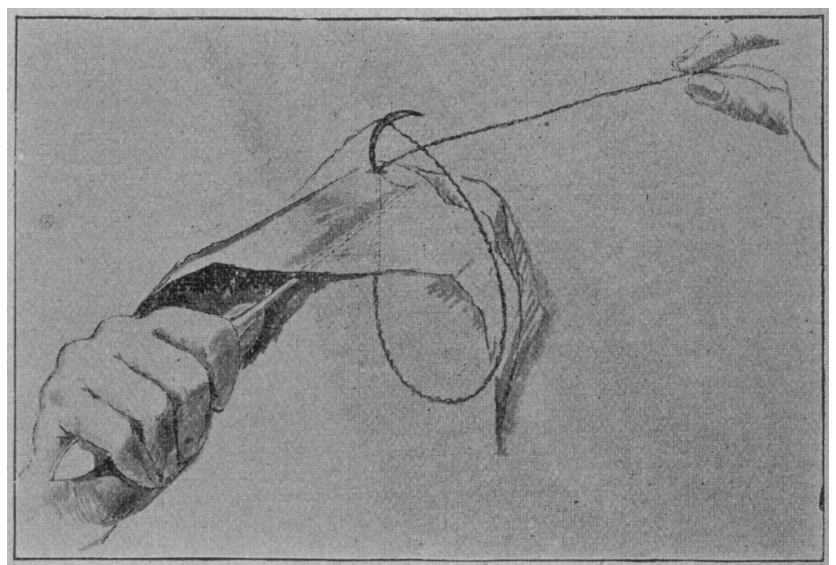

Figure 8.

Now withdraw the threaded needle from the pedicle, thereby forming a loop upon one side of the pedicle, the ends passing and crossing through the same opening appear upon the other side and may be tied,

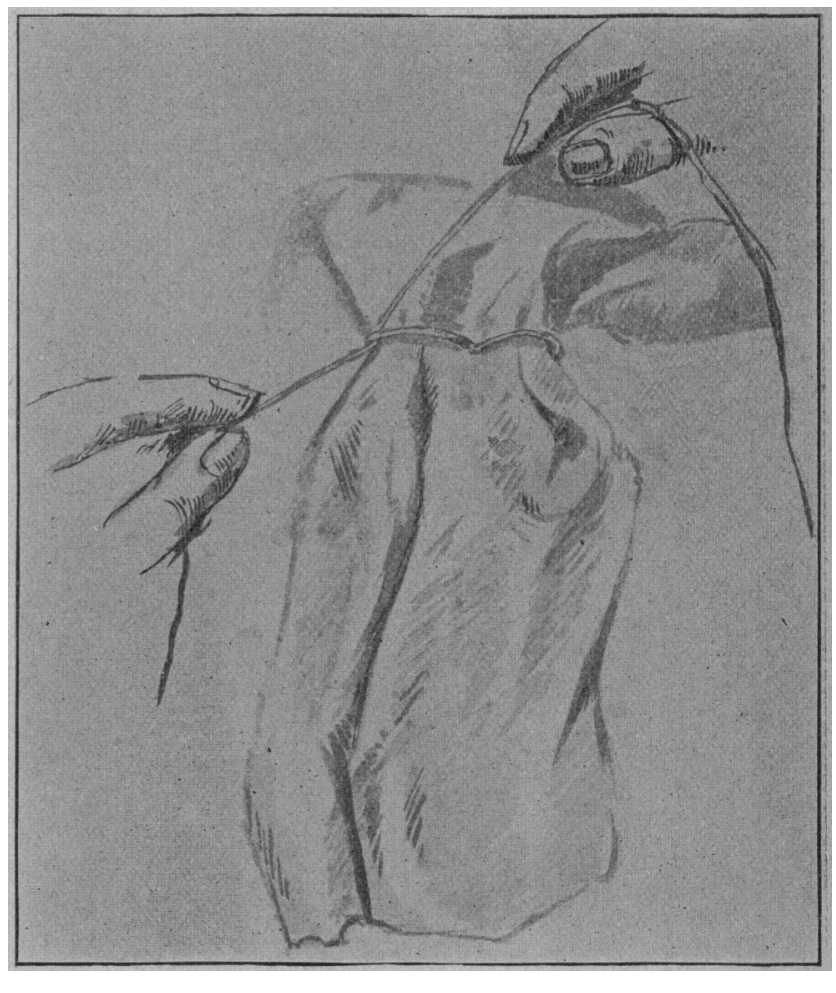

Figure 4.

as shown in Fig. 4, or, in a very broad pedicle that can not be securely tied with one figure-of-8, the needle may be passed through the pedicle at a distance of one-half inch or more, threaded with one of the free ends, then unthreaded of that end and again threaded: 
with the other one, and so on, until the entire pedicle is encompassed and the free ends tied.

307 Grand Avenue.

\section{A HANDY FORM OF HOT SNARE FOR TONSILLOTOMY. BY HENRY GRADLE, M.D. CHICAGO.}

It is acknowledged by many throat surgeons that the galvano-cautery snare is the best instrument for the removal of tonsils, because its acts very efficiently and prevents bleeding. Any one who has used either the hot or the cold snare for tonsillotomy has been able to observe that the wire loop slipped over the tonsil, after the latter has been pulled forward with a tenaculum or appropriate forceps, will grasp a larger portion of it than can be sliced off with any form of

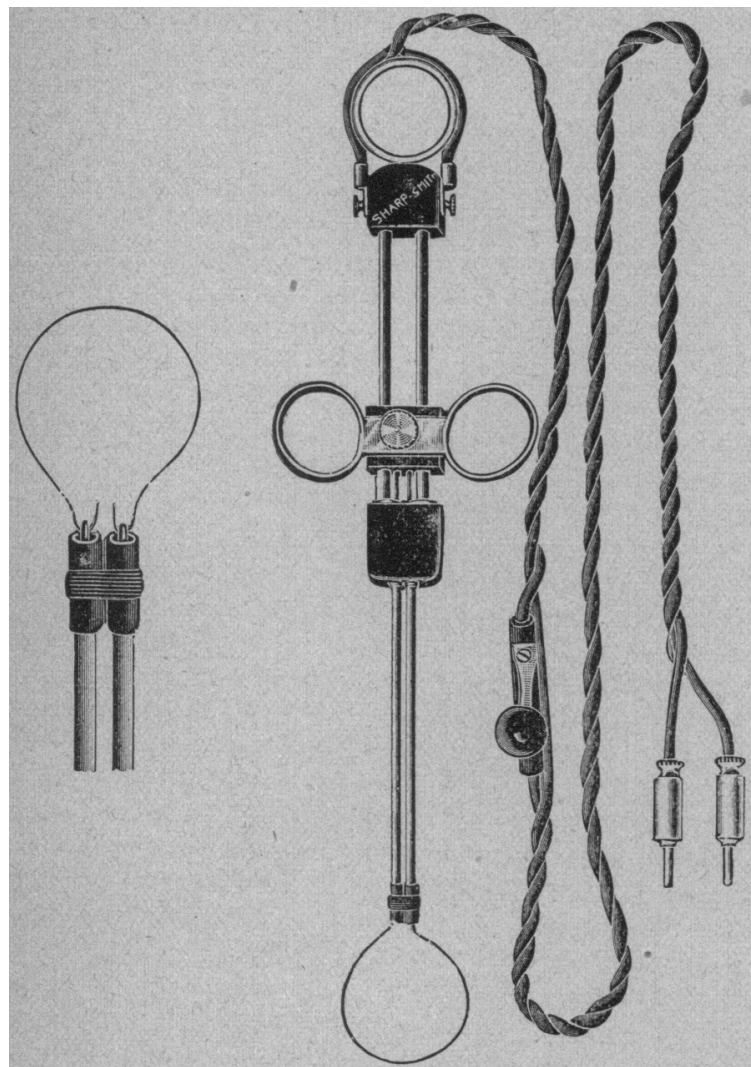

guillotine. But with slight experience the snare will be found even more thorough than the use of bistoury or scissors. The cold wire, however, is quite painful and with a Wyeth's snare it may even happen that the wire is pulled out of the stylet instead of cutting completely through the tonsil, if the gland is fibrous in consistency. The hot wire on the other hand cuts its way as easily as a oharp knife, even if only a dull red heat is employed. As this degree of heating is sufficient to stop all bleeding it is unnecessary to bring the wire to more than a dull red glow and indeed we only inflict more pain and produce a slower healing wound by heating the wire beyond this point. Since the current raises the wire progressively to a higher temperature as the loop gets shorter, it is best to press on the key and accordingly close the current in an intermittent fashion. The wound heals a little more slowly than a clean cut with the knife. But if the wire is not unnecessarily hot I find the wound entirely cicatrized in from five to ten days, according to the size, which is perhaps, two days more than for a corresponding tonsil cut with the knife.

The hot snare prevents bleeding entirely. If a few drops of blood appear they are due to unsuccessful grasping with the tenaculum. However trivial the bleeding may be in most tonsillotomies, anyone who has ever worked over one of the "exceptional" cases of tonsillar hemorrhage can appreciate the advantage of a bloodless operation. Even if fatal cases are very rare, annoying bleeding from tonsil cuts is not uncommon, especially in adults.

My reasons for devising a new instrument are the weakness of the ordinary snare canulæ and especially the loss of time incurred in wiring the hot snare as it is found in the market. I have hence designed a snare for tonsillotomy, which, however, can be used for any other purpose where straight and not very thin tubes are applicable. It consists of a handle made of two parallel brass bars, $11 \mathrm{~cm}$. long, mounted in rubber blocks at both ends. A third rubber block slides along the bars when guided by the fingers inserted through the rings on the block, while the thumb rests in the ring at the rear end of the handle. The sliding block holds two insulated steel stylets which when pushed forward, protrude through the two canulæ in front of the handle just far enough to allow a wire to be slipped through the eye in each stylet. The two canulæ, $9 \mathrm{~cm}$. long and $2.5 \mathrm{~mm}$. thick, are insulated by separation along their length and by a soft rubber tube slipped over the end, while their ends are strengthened by wire wound around the rubber insulation. Steel (piano) wire is a better material for the loop than platinum on account of its stiff. ness. The instrument can be used with a loop nearly $11 \mathrm{~cm}$. in circumference, but this size is rarely required. If a few suitable lengths of wire are prepared with their ends bent sharply, as shown in the accompanying cut, the burnt or softened wire can be replaced in a few seconds.

As shown in the figure the current enters the instrument through the cords permanently attached beside the rear ring, passes along the brass bars to the stylets directly through metallic contact in the front block as well as indirectly through the canulæ to the stylets and wire loop. Good connection is thus assured. I have not been able to devise a better place for the key which establishes the circuit than in the length of the cords twelve inches from the handle. Experience in over twenty-five operations has taught me that this arrangement is a practical one. The hand which seizes the tonsil with the tenaculum (put through the wire loop) presses the button as soon as the loop is in place.

The instrument has been made for me by Messrs. Sharp and Smith of this city.

\section{SELECTIONS.}

The Less Frequent Hazards of the Bicycle; Defects of the Tandem Wheel.-The editor of the Boston Medical and Surgical Journal, July 23, comments upon some of the less common accidents of 'cycling as follows :

"Altbough the worst casualties usually occur to riders going at high speed, there are certain conditions which render falls even when going at a low rate of speed serious and disfiguring. Of these the principal is that in a large number of cases, particularly those which are due to suddenly running into an obstacle, the weight of the head and body being carried 